\title{
Prenatal brain MRI of fetuses with Zika virus infection
}

\author{
Prisca Guillemette-Artur ${ }^{1}$ - Marianne Besnard ${ }^{2}$ • Dominique Eyrolle-Guignot ${ }^{3}$ • \\ Jean-Marie Jouannic ${ }^{4} \cdot$ Catherine Garel $^{5}$
}

Received: 15 March 2016 /Revised: 23 March 2016 / Accepted: 28 March 2016 / Published online: 18 April 2016

(C) Springer-Verlag Berlin Heidelberg 2016

\begin{abstract}
Background An outbreak of Zika virus was observed in French Polynesia in 2013-2014. Maternal Zika virus infection has been associated with fetal microcephaly and severe cerebral damage.

Objective To analyze the MRI cerebral findings in fetuses with intrauterine Zika virus infection.

Materials and methods We retrospectively analyzed prospectively collected data. Inclusion criteria comprised cases with (1) estimated conception date between June 2013 and May 2014, (2) available US and MRI scans revealing severe fetal brain lesions and (3) positive polymerase chain reaction for Zika virus in the amniotic fluid. We recorded
\end{abstract}

Catherine Garel

catherine.garel@aphp.fr

1 Service de Radiologie,

Centre Hospitalier de Polynésie Française,

Pirae, Tahiti, French Polynesia

2 Service de Réanimation Néo-natale, Centre Hospitalier de Polynésie Française, Pirae, Tahiti, French Polynesia

3 Service d'Obstétrique,

Centre Hospitalier de Polynésie Française,

Pirae, Tahiti, French Polynesia

4 Service de Médecine Fœtale, Hôpital d'Enfants Armand-Trousseau, Université Pierre et Marie Curie, Paris, France

5 Department of Radiology, Hôpital d'Enfants Armand-Trousseau, 26 Avenue du Dr Arnold Netter, Paris, France pregnancy history of Zika virus infection and analyzed US and MRI scans.

Results Three out of 12 cases of severe cerebral lesions fulfilled all inclusion criteria. History of maternal Zika virus infection had been documented in two cases. Calcifications and ventriculomegaly were present at US in all cases. MRI showed micrencephaly $(n=3)$, low cerebellar biometry $(n=2)$, occipital subependymal pseudocysts $(n=2)$, polymicrogyria with laminar necrosis and opercular dysplasia $(n=3)$, absent $(n=1)$ or hypoplastic $(n=1)$ corpus callosum and hypoplastic brainstem $(n=1)$.

Conclusion Severe cerebral damage was observed in our series, with indirect findings suggesting that the germinal matrix is the principal target for Zika virus. The lesions are very similar to severe forms of congenital cytomegalovirus and lymphocytic choriomeningitis virus infections.

Keywords Brain · Fetus - Intrauterine infection - Magnetic resonance imaging $\cdot$ Prenatal diagnosis $\cdot$ Ultrasound $\cdot$ Zika virus

\section{Introduction}

An outbreak of Zika virus was observed in French Polynesia, South Pacific, from October 2013 to April 2014. During this period, a mathematical model estimated that $86 \%$ of Polynesians were infected by the virus [1] and the seroprevalence of Zika virus was superior to $50 \%$ in adults and $66 \%$ in children [2]. This Zika virus epidemic was the largest ever documented [3] prior to the outbreak in Brazil and adjacent countries, which has been ongoing since June 2015. The link between maternal Zika virus infection and fetal microcephaly has recently been proved in the French Polynesian population [4]. Prenatal US findings have been reported recently in two 
cases in Brazil [5]; in a third Brazilian case, the mother underwent US and MRI [6]. The purpose of this study was to describe and analyze the MRI findings observed in the brain of fetuses diagnosed with Zika virus infection.

\section{Materials and methods}

This is a retrospective observational analysis of prospectively collected data conducted in the French Polynesia Hospital Center during the Zika virus epidemic from October 2013 to April 2014. We obtained formal approval from the ethics committee of the French Polynesia Hospital Center. Women with suspicion of fetal cerebral abnormality were referred to the prenatal diagnostic center of Tahiti. A second-line US examination was performed, using a Voluson Expert E8 US system (GE Healthcare, Waukesha, WI) with transabdominal or transvaginal probes, depending on fetal presentation. When biometric or morphological brain abnormalities were observed, MRI examination was offered and was performed on an Optima MR450w 1.5-T MR unit (GE Healthcare). Each MRI examination included T2-weighted sequences (singleshot fast spin echo [SSFSE] with repetition time/echo time [TR/TE] 960/80 ms and slice thickness $4 \mathrm{~mm}$ ) in three planes and T1-weighted sequences (spoiled gradient echo [SPGR] TR/TE $175 / 4.1 \mathrm{~ms}$, slice thickness $4 \mathrm{~mm}$ ) in at least one plane. In some cases, additional $\mathrm{T} 2 *$ sequences were performed. Pathological US and MRI scans were sent to Hospital Trousseau in Paris and reviewed by a pediatric radiologist (C.G.) with 25 years of experience in prenatal imaging.

In addition, amniocentesis was offered, and study of the amniotic fluid included karyotyping and polymerase chain reaction for cytomegalovirus. Retrospectively a few months later, in the same sample of amniotic fluid that was still available, polymerase chain reaction for Zika virus and lymphocytic choriomeningitis virus was also performed. In cases of severe fetal abnormalities, in accordance with French legislation and in agreement with the Multidisciplinary Prenatal Center of Hospital Trousseau, termination of pregnancy was performed at parental request.

The following data were recorded: pregnancy history (possible symptoms consistent with Zika virus infection such as erythema, fever, arthralgia and conjunctivitis), maternal serological status for toxoplasmosis and rubella, maternal body mass index, first-trimester US findings, gestational age at US detection of cerebral abnormalities, US and MRI findings and pregnancy outcome. Microcephaly was defined as head circumference (US measurements) below the 3rd centile. Micrencephaly was defined at MRI as cerebral biparietal diameter and fronto-occipital diameters below the 3rd centile [7].

In cases of pregnancy termination, placental samples were obtained and fetuses underwent clinical examination and radiography, but no fetus underwent autopsy, which is not available in French Polynesia.

We included all cases with estimated conception date between June 2013 and May 2014, available US and MRI scans showing severe fetal brain lesions, and positive polymerase chain reaction for Zika virus in the amniotic fluid. We excluded severe cerebral lesions diagnosed in the first trimester, cases with a single cerebral malformation and lesions with other known etiology.

\section{Results}

During the study period, 4,285 women delivered in the French Polynesia Hospital Center. Seven cases were excluded and comprised exencephaly $(n=1)$, transient mild ventriculomegaly $(n=1)$, corpus callosum agenesis $(n=3)$, posterior fossa cyst $(n=1)$ and congenital toxoplasmosis $(n=1)$. Twelve cases of severe fetal cerebral malformations were observed, either isolated $(n=6)$ or associated with other malformations $(n=6)$. Microcephaly was present in 7 fetuses. Karyotyping was performed in 11 cases and polymerase chain reaction for cytomegalovirus, lymphocytic choriomeningitis virus and Zika virus was performed in 8, 8 and 7 cases, respectively. Karyotype was normal in all cases and polymerase chain reaction for cytomegalovirus and lymphocytic choriomeningitis virus was negative. Polymerase chain reaction for Zika virus was positive in 4 cases. MRI was performed in 7 cases, including 3 with polymerase chain reaction positive for Zika virus. Termination of pregnancy was performed in 10 cases. The data are summarized in Fig. 1.

Three fetuses met the inclusion criteria. In two cases the mothers had symptoms consistent with Zika virus infection during the first trimester of pregnancy. In all cases, the mothers elected to terminate the pregnancy. The main data regarding these three cases are summarized in Table 1. Case numbers refer to this table.

In case 1, US demonstrated bilateral ventriculomegaly and parenchymal calcifications without microcephaly. At MRI, performed at 28.4 weeks of gestation (Fig. 2), the vermis was very small; the supratentorial space demonstrated occipital subependymal pseudocysts, ventriculomegaly, diffuse polymicrogyria, opercular dysplasia and abnormal cortical intensity consistent with laminar necrosis.

In case 2, US showed marked microcephaly, ventriculomegaly with large occipital subependymal pseudocysts, absent corpus callosum and cavum septi pellucidi and parenchymal calcifications. At MRI, performed at 31.1 weeks of gestation (Fig. 3), all these findings were confirmed and MRI also revealed associated brainstem and cortical abnormalities.

In case 3, US showed microcephaly, hypoplastic corpus callosum and diffuse parenchymal calcifications. MRI at 
Fig. 1 Diagram details the 12 fetuses with severe cerebral malformations observed in French Polynesia during the study period. No PCR no polymerase chain reaction for Zika virus was performed, $P C R$ polymerase chain reaction, $Z K V$ Zika virus, $Z K V+$ positive $\mathrm{PCR}$ for Zika virus, $Z K V$ - negative PCR for Zika virus

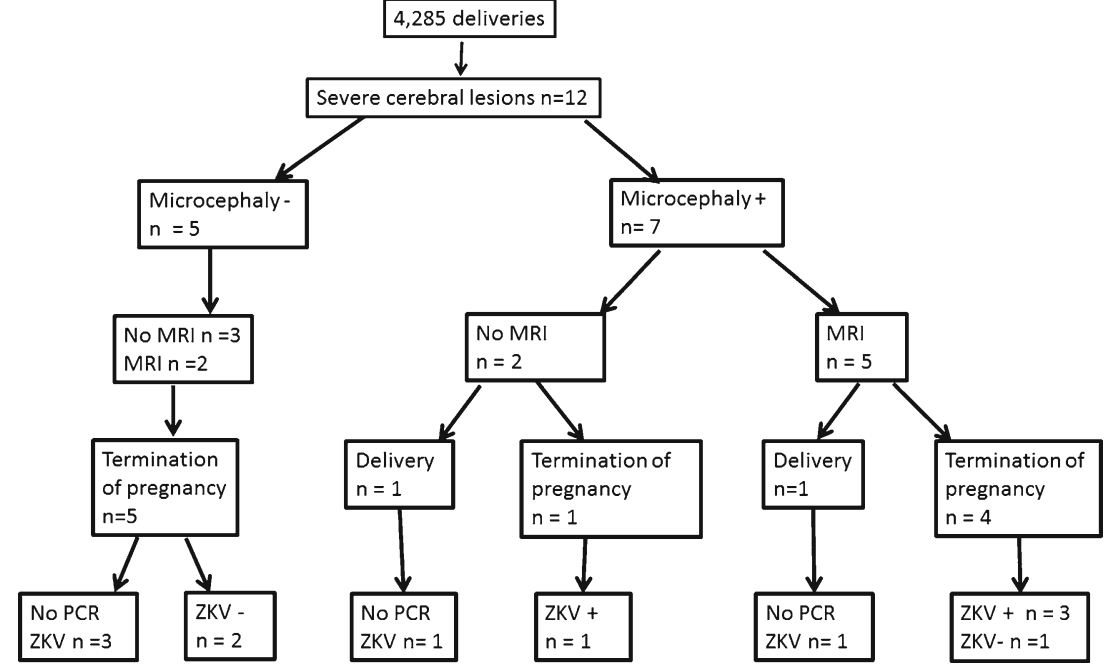

25 weeks of gestation (Fig. 4) confirmed these findings and also showed ventriculomegaly with diffuse polymicrogyria and opercular dysplasia.

\section{Discussion}

Zika virus is an arbovirus first isolated in 1947 in the Zika forest of Uganda [8]. Zika virus epidemics have been reported in Gabon in 2007, on the island of Yap (Pacific Ocean) in 2007 and in French Polynesia in 2013-2014 [3], and there is currently a large epidemic in Brazil and adjacent countries. Zika virus is mostly transmitted by mosquitoes [3]. There is also concern for potential sexual transmission [9]. The possibility of maternofetal transmission was first suggested in two Polynesian newborns [10], and vertical transmission to the fetus has been recently documented in Brazil $[5,6]$.

About 270,000 people live in French Polynesia and 4,200 births are recorded per year, with $0-1$ annual cases of microcephaly. Cases of microcephaly dramatically increased during the 2013-2014 Zika virus epidemic, and the risk of fetal microcephaly following first-trimester Zika virus infection has been quantitatively estimated at $95 / 10,000$, without ruling out possible increased risk of microcephaly following second- or third-trimester infection [4].

Cerebral US findings have been recently reported in three fetuses in Brazil. In these cases, the mothers had symptoms related to Zika virus infection, and positive polymerase chain reaction for Zika virus was found in the amniotic fluid in two cases [5], and retrospective assessment of maternal Zika virus infection was found in the third case [6]. All three fetuses showed microcephaly and parenchymal calcifications. Moreover, US depicted corpus callosum dysgenesis with additional vermian dysgenesis in one fetus and ventriculomegaly and brainstem thinning in another [5].
In our series, 1/3 fetuses (case 1) did not show microcephaly at US. However, MRI revealed marked micrencephaly with very low cerebral biometric parameters and enlarged pericerebral space. In one fetus (case 2), the transverse cerebellar diameter was below the lower limit, with enlarged vermian primary fissure and absent bulge of the pons. Case 1 showed a small vermis. Ventriculomegaly was observed in all three cases and, interestingly, it was associated twice (cases 1 and 2) with large occipital subependymal pseudocysts. In all cases, diffuse polymicrogyria was observed, in association with opercular dysplasia and calcifications and twice with markedly thinned cortex. In two cases (2 and 3), the corpus callosum was abnormal. All these findings show similarities to the findings reported in Brazil [5]. However, occipital pseudocysts and cortical anomalies were not described in two Brazilian cases [5]. Analysis of the MR sagittal image provided in the third Brazilian case [6] reveals the presence of an occipital pseudocyst. In our study, there is no doubt that MRI helped in detecting polymicrogyria, laminar necrosis and brainstem anomalies. The contribution of MRI in this field is well established [11]. In our series, maternal obesity was an additional factor, accounting for the discrepancy between US and MRI findings in one case [12].

Some of the cerebral abnormalities observed in these three fetuses resemble lesions commonly observed in other intrauterine infections. Of note, none of these fetuses demonstrated associated intrauterine growth retardation. Additionally, ventriculomegaly is a very common and nonspecific finding in most congenital infections.

Subependymal pseudocysts are located in the subependymal area surrounding the lateral ventricles. They are commonly observed before and after birth, and most of them carry a favorable prognosis. However they can also be present in the setting of pathological conditions including infectious intrauterine diseases. It has been recently underlined that certain features of 


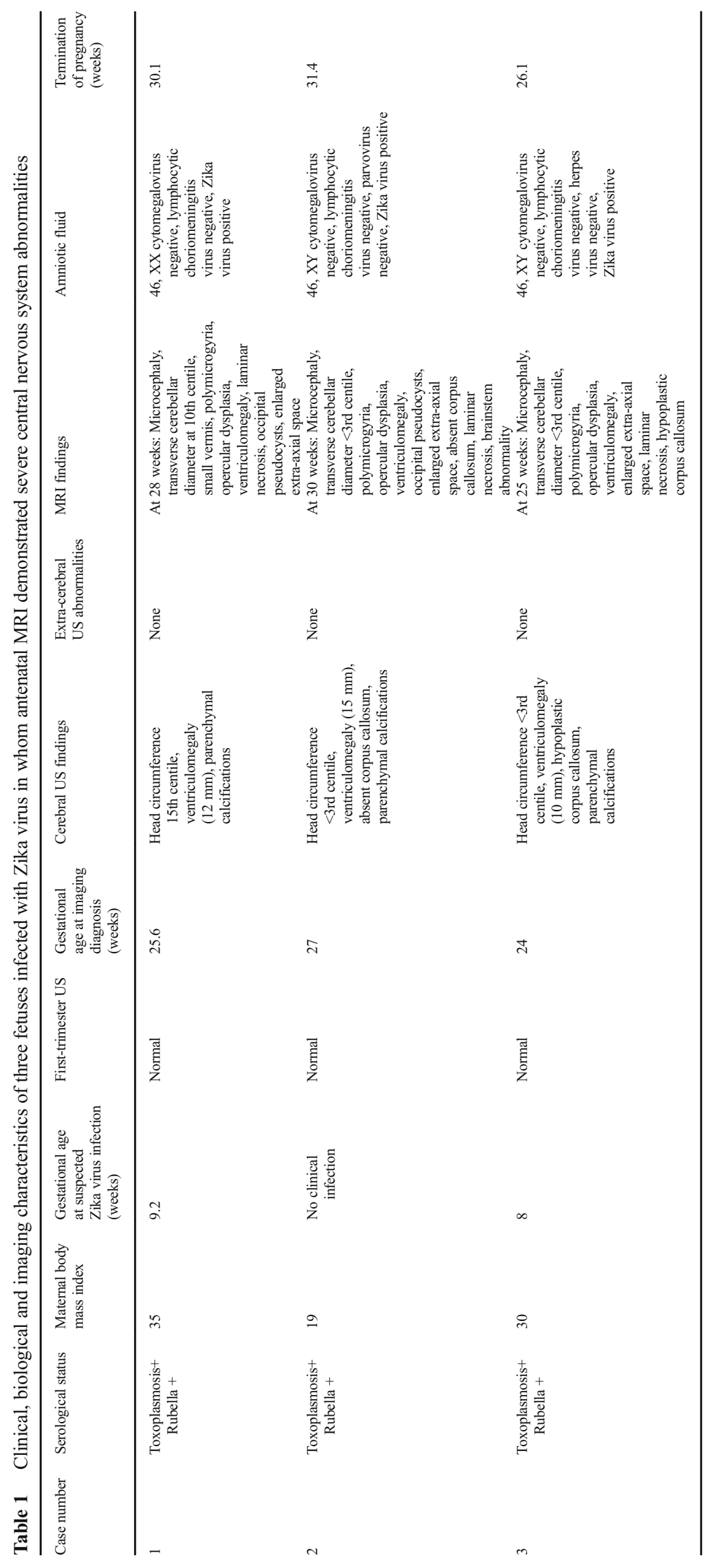


Fig. 2 Case 1. Brain MRI at 28 weeks of gestation. a-d T2weighted sagittal (a, b) and coronal (c), and T1-weighted coronal (d) slices. The vermis is small and does not demonstrate normal morphology. The bulge of the pons is present. Both lateral ventricles are dilated, with visibility of large occipital subependymal pseudocysts (arrow in b). There is bilateral opercular dysplasia. The cortex is irregular (arrowhead in c), which suggests diffuse polymicrogyria. T2-hypointense and T1hyperintense areas (dotted arrows in $\mathbf{c}$ and $\mathbf{d}$ ) of the cortex are consistent with calcifications in the setting of laminar necrosis
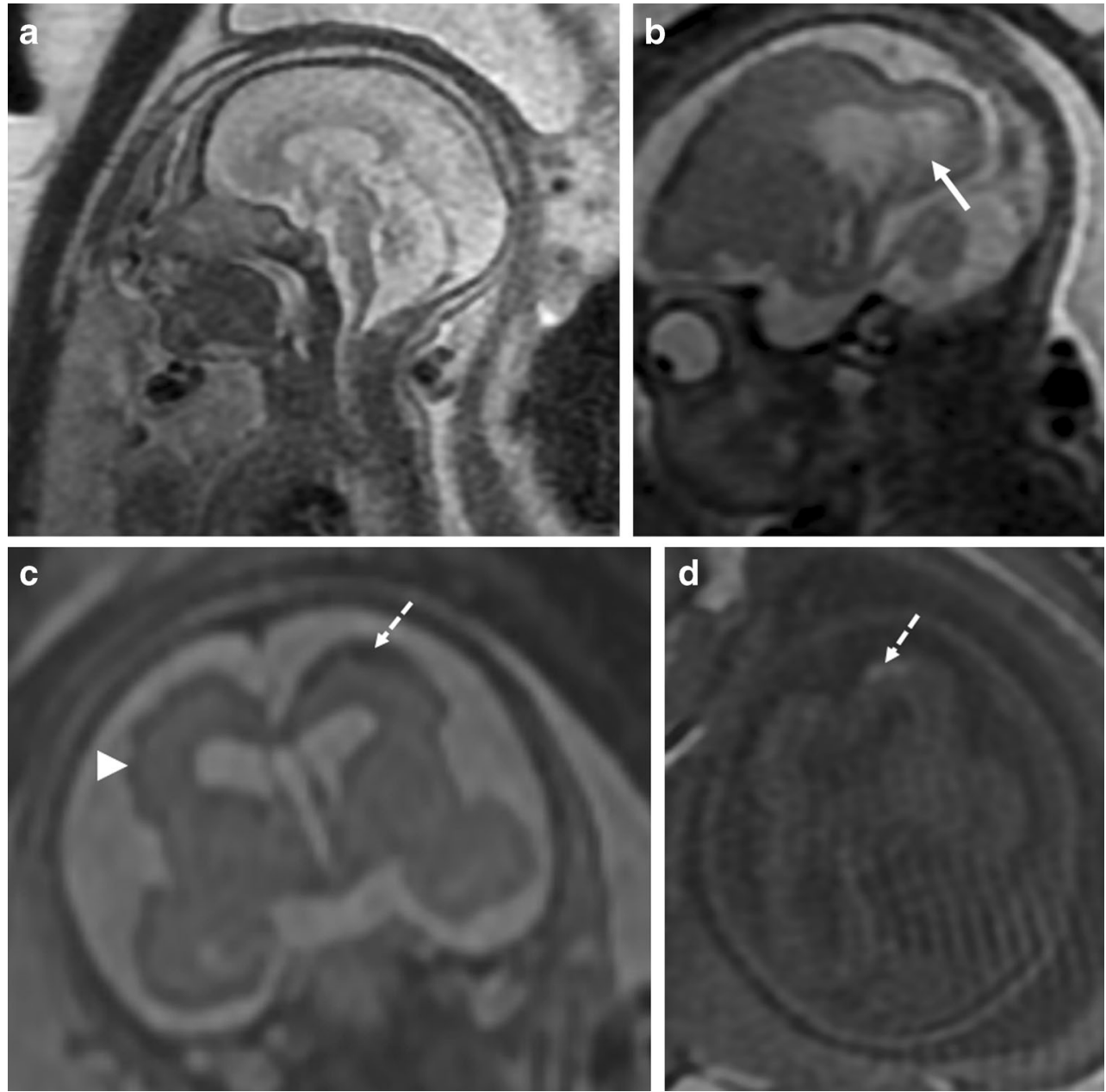

apparently isolated pseudocysts may indicate underlying pathology. In this study, the occipital localization was never observed in fetuses with normal outcome, while it was observed in the setting of cytomegalovirus infection [13]. Such a pattern has also been reported as being one of the characteristic findings of fetal cerebral lesions resulting from cytomegalovirus infection [14]. It is ascribed to germinal matrix necrosis; indeed, neuronal precursor cells, initially located in the germinal matrix, have been proved to be the principal targets for cytomegalovirus [15]. Our cases indicate that occipital subependymal pseudocysts can also result from intrauterine Zika virus infection.

Destruction of the germinal matrix results in microcephaly and cortical development disorders, accounting for diffuse polymicrogyria, laminar necrosis and gyration abnormalities. These findings are commonly observed in severe forms of cytomegalovirus infection. Laminar necrosis appears hyperintense in $\mathrm{T} 1-\mathrm{W}$ MR images, which is attributed to microcalcifications and also accounts for $\mathrm{T} 2$ hyperintensity of these lesions [16]. This was observed in the three fetuses in our series.
Similarly, lymphocytic choriomeningitis virus - a member of the Arenaviridae family of viruses using rodents as its principal reservoir - has also exhibited a strong tropism for neuroblasts. Congenital lymphocytic choriomeningitis virus infection can lead to microcephaly, periventricular calcifications, cortical dysplasia and focal cerebral destruction. This can mimic severe cerebral damage from cytomegalovirus [17].

The precise effect of Zika virus on the developing brain has not been widely studied yet; similarities of lesions observed in cytomegalovirus, lymphocytic choriomeningitis virus and Zika virus infection suggest that Zika virus might also predominantly affect neural precursor cells. A recent experimental model system has shown that Zika virus infection attenuates growth of human neural progenitor cells [18]. Polymicrogyria has also been reported as a result of intrauterine herpes simplex virus infection [19].

Parenchymal calcifications are a hallmark of most congenital infections and have been reported in association with cytomegalovirus [14], lymphocytic choriomeningitis virus [17], herpes simplex virus infection [19] and varicella-zoster virus [20]. In toxoplasmosis, fetuses usually demonstrate cerebral 
Fig. 3 Case 2. Brain MRI at 30 weeks of gestation. a-d T2weighted sagittal $(\mathbf{a}, \mathbf{b})$ and coronal (c), and T1-weighted axial (d) slices are shown. The bulge of the pons is not visible on the midline sagittal slice (a) and the primary fissure (arrow) is too wide. The supratentorial space is very small and the pericerebral space is enlarged. The occipital subependymal pseudocysts are well visible (arrow in b). The corpus callosum and the cavum septi pellucidi are not seen. Note the bilateral opercular dysplasia and a very thin and irregular cortex, consistent with diffuse polymicrogyria. The normal sulcation is absent. In some areas, marked cortical T2 hypointensity (dotted arrow in c) and $\mathrm{T} 1$ hyperintensity (arrowhead in d) are suggestive of calcifications
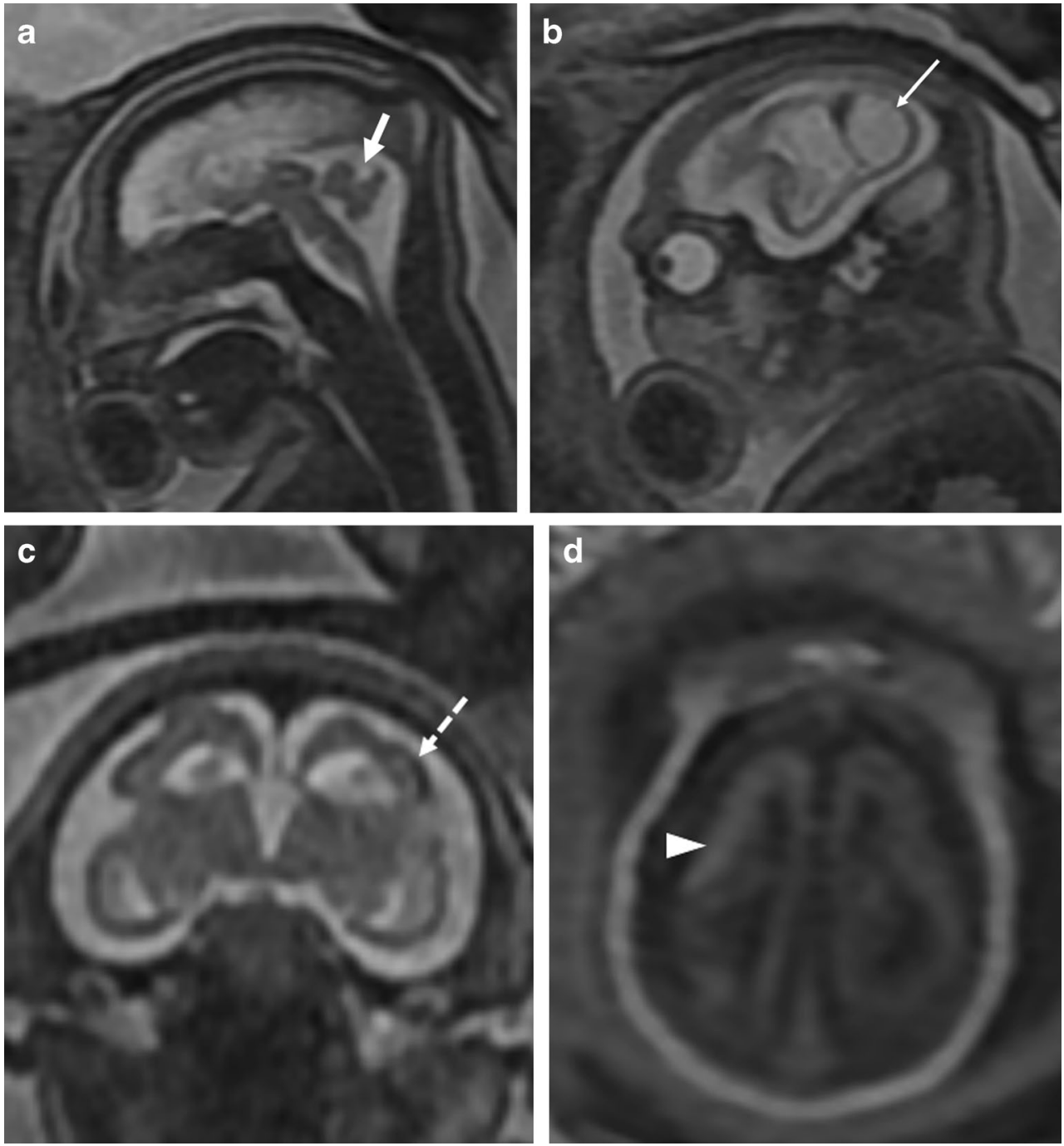

parenchymal echogenic nodules occurring in multiple areas without predilection for the periventricular zone [21]. In our study, the three fetuses presented with multiple parenchymal calcifications, which were in fact better demonstrated using US than MRI, as usually observed.

Corpus callosum abnormalities were present in two cases and consisted of either absence or marked hypoplasia of this structure. These findings are similar to those described in two cases from Brazil [5] and are most likely of disruptive origin, resulting from massive neuronal loss and cerebral damage from Zika virus.

Our study shows that both brainstem and cerebellum might also be affected by Zika virus. Two fetuses showed cerebellar hypoplasia and in one case the brainstem was thinned without visibility of the bulge of the pons. These findings are similar to the description of two fetuses in Brazil [5]. Cytomegalovirus might also affect the cerebellum, causing hypoplasia and hemorrhage [14]. Similarly, cerebellar hypoplasia is reported in congenital lymphocytic choriomeningitis virus infection [17], while parvovirus B19 infection might be responsible for cerebellar hemorrhage [22]. Brainstem hypoplasia might result from profound cerebellar damage. Another cause might be that the brainstem constitutes a specific target of Zika virus.

In two of our three cases, maternal infection occurred during the first trimester. In a recent article [23], two cases of microcephaly and two additional cases of brain calcifications were observed following a second-trimester Zika virus maternal infection. It is likely that in the future, as it is observed for cytomegalovirus, less severe cerebral lesions might be described when maternal infection occurs late during pregnancy.

\section{Conclusion}

This small series analyzes cerebral lesions observed in congenital Zika virus infection using fetal MRI. As in the few other reported cases in the literature, severe cerebral damage was observed in our series. The findings we observed are in 
Fig. 4 Case 3. Brain MRI at 25 weeks of gestation. a-d T2weighted sagittal (a) and coronal (b, c), and T1-weighted coronal (d) slices are shown. The morphology of the posterior fossa structures is normal. The supratentorial space is small, with enlarged pericerebral space. The corpus callosum (arrowheads in a and $\mathbf{b}$ ) is very thin. The ventricles are enlarged and the cortex is very thin and irregular, accounting for diffuse polymicrogyria. Note the bilateral opercular dysplasia and diffuse laminar necrosis with $\mathrm{T} 2$ hypointensity/T1 hyperintensity (dotted arrows) of the cortex
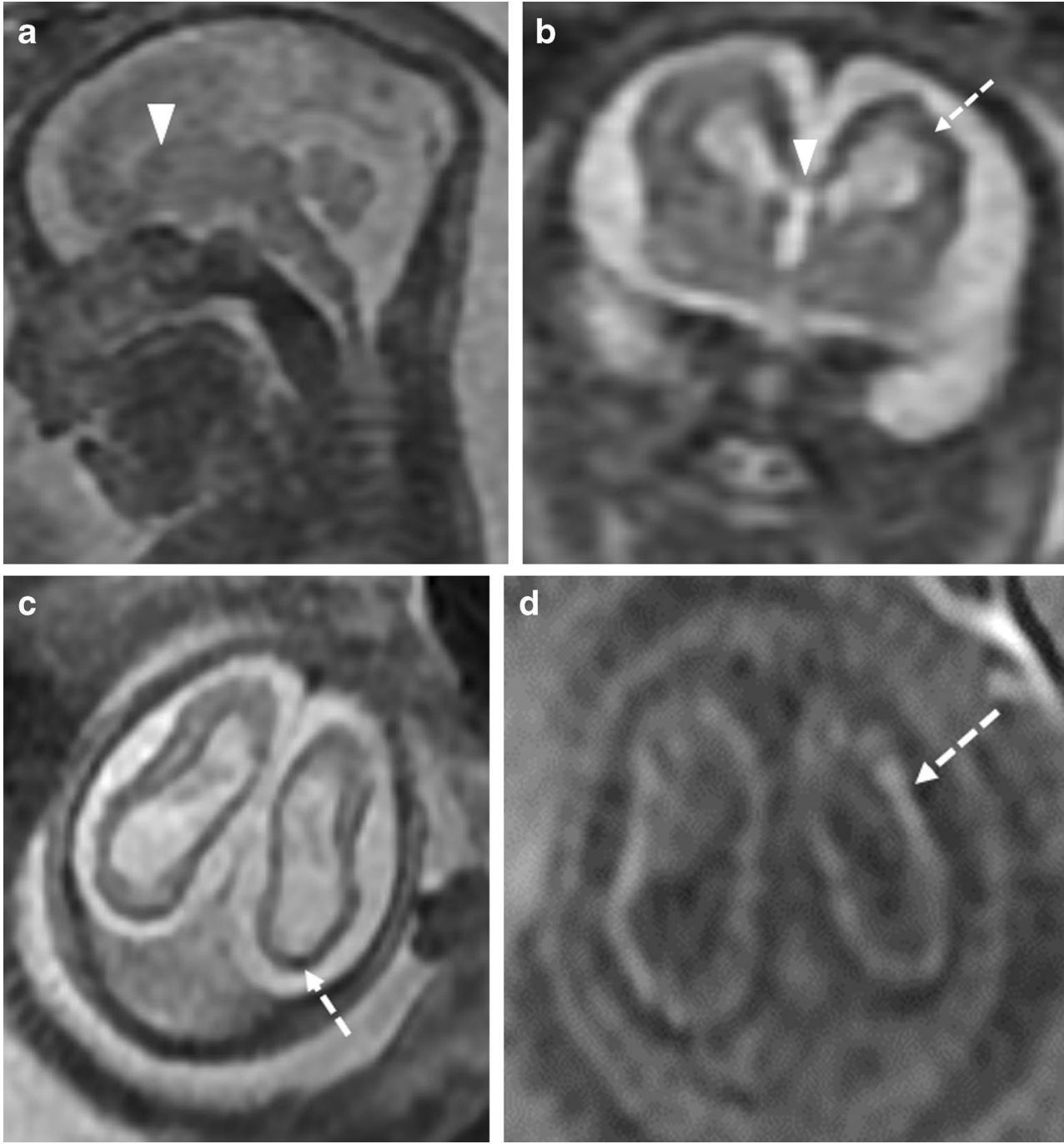

accordance with very recent experiments showing that neural precursor cells constitute a direct target for Zika virus. Most probably, as has been observed in cases of cytomegalovirus, less severe cerebral lesions might result from maternal infection occurring later during pregnancy.

\section{Compliance with ethical standards}

\section{Conflicts of interest None}

\section{References}

1. Kucharski AJ, Funk S, Eggo RM et al (2016) Transmission dynamics of Zika virus in island populations: a modelling analysis of the 2013-2014 French Polynesia outbreak. bioRxiv. doi:10.1101/ 038588

2. Cao-Lormeau VM, Roche C, Teissier A et al (2014) Zika virus, French Polynesia, South Pacific, 2013. Emerg Infect Dis 20: $1085-1086$
3. Ioos S, Mallet HP, Leparc Goffart I et al (2014) Current Zika virus epidemiology and recent epidemics. Med Mal Infect 44:302-307

4. Cauchemez S, Besnard M, Bompard P et al (2016) Association between Zika virus and microcephaly in French Polynesia, 20132015: a retrospective study. Lancet. doi:10.1016/S0140-6736(16) 00651-6

5. Oliveira Melo AS, Malinger G, Ximenes R et al (2016) Zika virus intrauterine infection causes fetal brain abnormality and microcephaly: tip of the iceberg? Ultrasound Obstet Gynecol 47:6-7

6. Werner H, Fazecas T, Guedes B et al (2016) Intrauterine Zika virus infection and microcephaly: perinatal imaging correlations with 3D virtual physical models. Ultrasound Obstet Gynecol. doi:10.1002/ uog. 15901

7. Tilea B, Alberti C, Adamsbaum C et al (2009) Cerebral biometry in fetal magnetic resonance imaging: new reference data. Ultrasound Obstet Gynecol 33:173-181

8. Dick GW, Kitchen SF, Haddow AJ (1952) Zika virus. I. Isolations and serological specificity. Trans R Soc Trop Med Hyg 46:509-520

9. Musso D, Roche C, Robin E et al (2015) Potential sexual transmission of Zika virus. Emerg Infect Dis 21:359-361

10. Besnard M, Lastere S, Teissier A et al (2014) Evidence of perinatal transmission of Zika virus, French Polynesia, December 2013 and February 2014. Euro Surveill 19(13) 
11. Blondiaux E, Garel C (2013) Fetal cerebral imaging — ultrasound vs. MRI: an update. Acta Radiol 54:1046-1054

12. Paladini D (2009) Sonography in obese and overweight pregnant women: clinical, medicolegal and technical issues. Ultrasound Obstet Gynecol 33:720-729

13. Esteban H, Blondiaux E, Audureau E et al (2015) Prenatal features of isolated subependymal pseudocysts associated with adverse pregnancy outcome. Ultrasound Obstet Gynecol 46:678-687

14. Malinger G, Lev D, Lerman-Sagie T (2011) Imaging of fetal cytomegalovirus infection. Fetal Diagn Ther 29:117-126

15. Mutnal MB, Cheeran MC, Hu S et al (2011) Murine cytomegalovirus infection of neural stem cells alters neurogenesis in the developing brain. PLoS One 6:e16211

16. Garel C, Delezoide AL, Elmaleh-Berges M et al (2004) Contribution of fetal MR imaging in the evaluation of cerebral ischemic lesions. AJNR Am J Neuroradiol 25:1563-1568

17. Bonthius DJ (2012) Lymphocytic choriomeningitis virus: an underrecognized cause of neurologic disease in the fetus, child, and adult. Semin Pediatr Neurol 19:89-95
18. Tang H, Hammack C, Ogden SC et al (2016) Zika virus infects human cortical neural progenitors and attenuates their growth. Cell Stem Cell. doi:10.1016/j.stem.2016.02.016

19. Duin LK, Willekes C, Baldewijns MM et al (2007) Major brain lesions by intrauterine herpes simplex virus infection: MRI contribution. Prenat Diagn 27:81-84

20. Mandelbrot L (2012) Fetal varicella — diagnosis, management, and outcome. Prenat Diagn 32:511-518

21. Malinger G, Werner H, Rodriguez Leonel JC et al (2011) Prenatal brain imaging in congenital toxoplasmosis. Prenat Diagn 31: 881-886

22. Glenn OA, Bianco K, Barkovich AJ et al (2007) Fetal cerebellar hemorrhage in parvovirus-associated non-immune hydrops fetalis. J Matern Fetal Neonatal Med 20:769-772

23. Brasil P, Pereira JP Jr, Raja Gabaglia C et al (2016) Zika virus infection in pregnant women in Rio de Janeiro - preliminary report. N Engl J Med. doi:10.1056/NEJMoa1602412 